\title{
The role of fluid viscosity in an immersed granular collapse
}

\author{
Geng Chao Yang ${ }^{1}$, Chung Yee Kwok ${ }^{1, \star}$, and Yuri Dumaresq Sobral ${ }^{2}$ \\ ${ }^{1}$ Department of Civil Engineering, The University of Hong Kong, Haking Wong Building, Pokfulam Road, Hong Kong \\ ${ }^{2}$ Departamento de Matemática, Universidade de Brasília, Campus Universitário Darcy Ribeiro, 70910-900 Brasília, DF, Brazil
}

\begin{abstract}
Instabilities of immersed slopes and cliffs can lead to catastrophic events that involve a sudden release of huge soil mass. The scaled deposit height and runout distance are found to follow simple power laws when a granular column collapses on a horizontal plane. However, if the granular column is submerged in a fluid, the mobility of the granular collapse due to high inertia effects will be reduced by fluid-particle interactions. In this study, the effects of fluid viscosity on granular collapse is investigated qualitatively by adopting a numerical approach based on the coupled lattice Boltzmann method (LBM) and discrete element method (DEM). It is found that the granular collapse can be dramatically slowed down due to the presence of viscous fluids. For the considered granular configuration, when the fluid viscosity increases. the runout distance decreases and the final deposition shows a larger deposit angle.
\end{abstract}

\section{Introduction}

Granular flows which are saturated by or immersed in fluids are ubiquitous phenomena in nature and industries, such as debris flow, submarine avalanche, food manufacturing and pharmacy. It is rather important to investigate these hydrogranular flows because of their crucial roles in sediment transport, shaping the landscape, risk assessment and industrial optimization. However, complex interactions between granular materials and fluids are yet to be fully understood.

Dry gravitational flows have been widely studied experimentally by a sudden release of granular column on a horizontal plane (see Fig. 1) over the last decades, focusing on flow kinematics and deposit morphologies [1,2]. Scaling laws relating the deposit height and runout distance to the initial aspect ratio $\left(h_{0} / x_{0}\right)$ of the granular column are proposed. As an important parameter in engineering and geophysics, the runout distance generally increases when the initial aspect ratio increases.

However, for granular flows in a viscous fluid, the runout distance also depends on other physical parameters, such as the initial volume fraction [3]. When the initial particle volume fraction is smaller than a critical value equal to 0.58 , the scaled runout distance is longer compared to dry flows because of the generated excess pore pressure due to fluid expulsion [3]. In contrast, flows with high initial particle volume fraction travel similar distance as dry flows.

Apart from the significance of immersed granular collapse, the mechanisms and influence of fluids on flow kinematics and deposit morphologies are yet to be fully understood. The focus of this study is to investigate the role

\footnotetext{
^e-mail: fkwok8@hku.hk
}

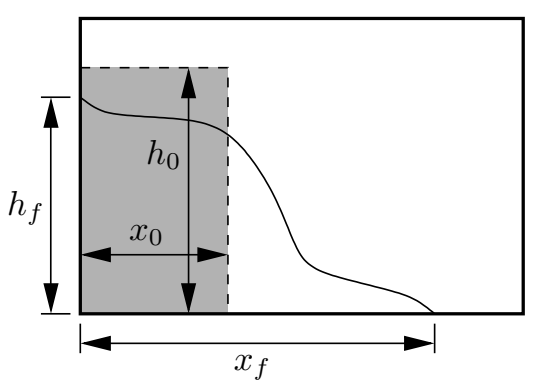

Figure 1. Granular Collapse with initial column (dashed line) of height $h_{0}$ and length $x_{0}$, and deposit of height $h_{f}$ and length $x_{f}$

of fluid viscosity on granular collapse in a fluid using the coupled lattice Boltzmann method (LBM) and discrete element method (DEM).

\section{Methodology}

LBM has been developed to become an alternative tool to traditional computational fluid dynamics (CFD) to couple with DEM, benefiting from the direct calculation of the drag force without ad hoc models [4-7]. LBM is a mesoscopic description of fluid flow based on the kinetic theory. Statistically, fluid behavior at macroscopic scale is nothing but the average micro-dynamics of fluid molecules. Meanwhile, macroscopic fluid behavior is also insensitive to the detailed dynamics of individual molecules. Therefore, LBM is able to describe the fluid system by a collection of molecules residing on a regular lattice. A set of density distribution functions are used to represent the collection of molecules at each lattice node, where collision between 
"fluid" molecules takes place by following the conservation laws of mass, momentum and energy. At each time step, the post-collision density distribution functions are passed to the neighboring lattice nodes by a streaming process.

In LBM, the evolution equation for the density distribution function $f_{i}(\mathbf{x}, t)$ with a BGK approximation [8], can be written as:

$$
f_{i}\left(\mathbf{x}+\mathbf{c}_{i} \delta_{t}, t+\delta_{t}\right)-f_{i}(\mathbf{x}, t)=-\frac{1}{\tau}\left[f_{i}(\mathbf{x}, t)-f_{i}^{e q}(\mathbf{x}, t)\right]
$$

where the density distribution function $f_{i}$ is related to the number of molecules at time $t$ positioned at $\mathbf{x}$ moving with velocity $c_{i}$ along the $i$ th direction at each lattice node. The time step for evolution and the relaxation time are $\delta_{t}$ and $\tau$, respectively. The equilibrium distribution function (EDF) is $f_{i}^{e q}$. Based on the conservation of mass and momentum, the fluid density $\rho$ and velocity $\mathbf{u}$ can be easily reconstructed from the velocity moments of the discrete density distribution functions:

$$
\begin{gathered}
\rho=\sum_{i} f_{i} \\
\rho \mathbf{u}=\sum_{i} \mathbf{c}_{i} f_{i}
\end{gathered}
$$

The pressure field $p$ is related to the fluid density by the equation of state:

$$
p=c_{s}^{2} \rho
$$

The lattice speed of sound is $c_{s}$ and equal to $1 / \sqrt{3}$ for the D3Q19 lattice arrangement [9].

While the fluid is simulated using LBM, DEM is adopted to take care of the solid particles. DEM has been widely used in granular flow applications [10-12]. The particle-particle collisions are governed by the Hertzian contact laws [13]. By considering the gravity $(\mathbf{G})$, contact forces $\left(\mathbf{F}_{c}\right.$ and $\left.\mathbf{T}_{c}\right)$ and hydrodynamic forces and torques $\left(\mathbf{F}_{f}\right.$ and $\mathbf{T}_{c}$ ), the positions and velocities of particles can be updated according to the Newton's second law:

$$
m \mathbf{a}=\mathbf{F}_{c}+\mathbf{F}_{f}+\mathbf{G}
$$

$$
\mathbf{I} \dot{\omega}=\mathbf{T}_{c}+\mathbf{T}_{f}
$$

The mass and moment of inertia of particles are $m$ and I, respectively. The acceleration is a and the angular velocity is $\omega$. The coupling between LBM and DEM is achieved by the immersed moving boundary method, initially proposed by Nobel and Torczynski [14]. For each cycle of LBM calculation, 100 sub-cycles of DEM calculation are conducted. During the sub-cycling process, the positions of DEM particles on the LBM lattice grid and the corresponding hydrodynamic forces and torques are not updated.
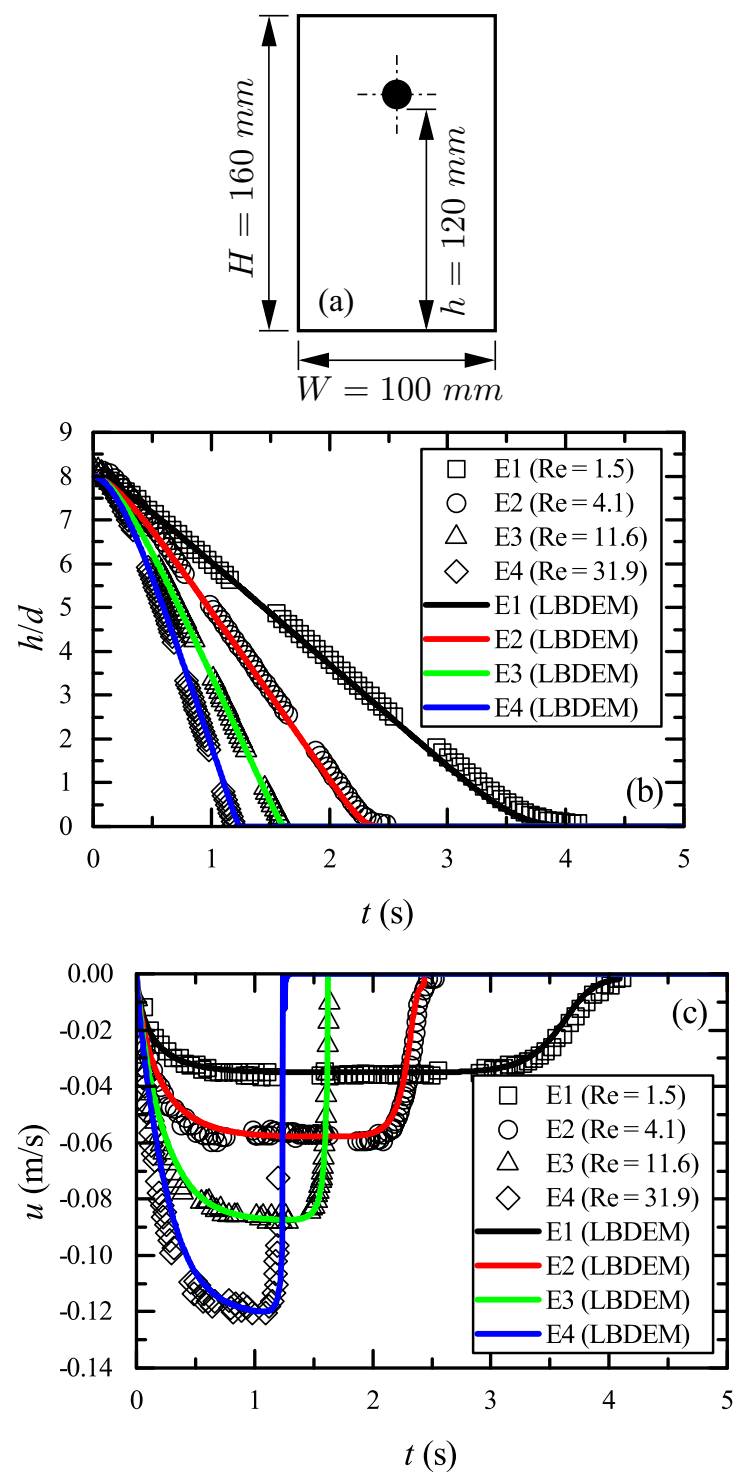

Figure 2. Single particle sedimentation test: (a) sketch of the experimental and numerical setup; (b) comparison between measured and simulated particle settling trajectory; (c) comparison between measured and simulated particle settling velocity

\section{Results and discussions}

\subsection{Single particle sedimentation test}

Fig. 2(a) shows the sketch of the experimental and numerical setups for the single particle sedimentation test conducted in [15]. A $15 \mathrm{~mm}$ diameter $(d)$ particle is released at a height $\left(h_{0}\right)$ equal to $120 \mathrm{~mm}$ with zero initial velocity within a container with dimension $100 \times 100 \times 160 \mathrm{~mm}$. During sedimentation of the particle, the settling velocity first increases until a terminal velocity $(u)$ is reached, at which the downward self-weight is balanced by the upward buoyancy force and drag force. We have repeated the same experiments referenced in [15] for four different values of Reynolds number (Re). The evolutions of particle trajectory and settling velocity from numerical simulations are compared to available experimental data in Fig. 2(b) and Fig. 2(c), respectively. It is shown that the LBM-DEM 

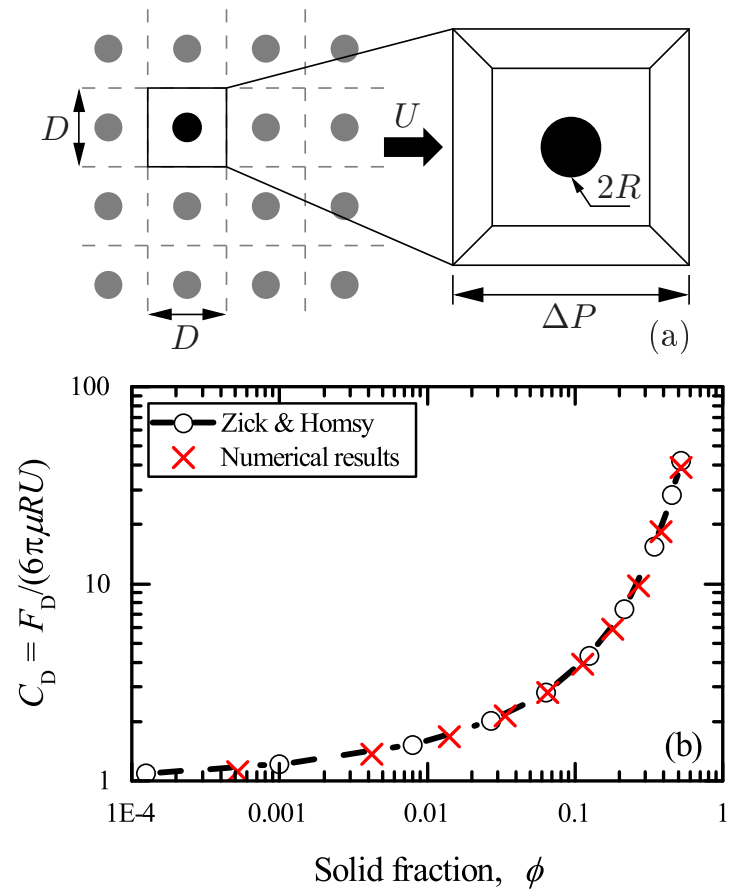

Figure 3. Flow through a three-dimensional periodic array of particles: (a) sketch of the numerical model; (b) comparison between numerical results and Zick \& Homsy's solution for the drag coefficients at various solid fractions

coupling scheme presented in this study can capture well the fluid-particle interactions in the single particle sedimentation test.

\subsection{Flow through a periodic array of particles}

Fig. 3(a) shows the sketch of a viscous flow through threedimensional periodic array of particles. In numerical simulations, only one cubic cell with size $D$ equal to $0.01 \mathrm{~m}$ is considered with periodic boundary conditions defined in all faces. A small pressure gradient $0.001 \mathrm{~Pa} / \mathrm{m}$ is applied to drive the flow from the left to the right. The simulations are run until the steady state is achieved at which point the flow velocity becomes steady. In order to get the drag forces at various solid fractions $\phi$, the particle radius is changed from $0.0005 \mathrm{~m}$ to $0.005 \mathrm{~m}$. Fig. 3(b) compares the normalized hydrodynamic forces from numerical simulations and Zick and Homsy's analysis [16], showing an excellent agreement over the entire range of solid fractions considered in this study.

\subsection{Granular collapse}

A sample of a granular column is first prepared using the gravitational deposition method. In this study, a rather dense initial packing is achieved by setting the particle friction coefficient to be zero, which is later adjusted to 0.4 before releasing the granular column. The other modelling parameters are summarized in Table 1.

In DEM, rigid walls are applied at all faces for the three-dimensional simulation domain. In LBM, non-slip
Table 1. Modelling parameters

\begin{tabular}{lll}
\hline & Parameters & Values \\
\hline Particle & Diameter, $d_{p}(\mathrm{~m})$ & 0.002 \\
& Density, $\rho_{p}\left(\mathrm{~kg} / \mathrm{m}^{3}\right)$ & 2500 \\
& Young's modulus, $E(\mathrm{~Pa})$ & $5 \mathrm{E} 6$ \\
& Poisson's ratio, $v$ & 0.35 \\
& Coefficient of restitution, $e$ & 0.5 \\
\hline Fluid & Density, $\rho_{f}\left(\mathrm{~kg} / \mathrm{m}^{3}\right)$ & 1000 \\
& Viscosity, $\mu_{f}(\mathrm{~Pa} \cdot \mathrm{s})$ & $0.01-0.05$ \\
\hline Granular & Initial width, $x_{0}(\mathrm{~m})$ & 0.02 \\
column & Aspect ratio, AR & 2.0 \\
& Initial volume fraction, $\phi$ & 0.62 \\
\hline
\end{tabular}

boundary condition is defined at the walls following the bounce-back rule [4]. In this study, the influence of viscosity on the granular collapse in a fluid is considered. As the granular column collapses onto the horizontal plane (see Fig. 1), the particles will push the fluid forward at the lower part of the simulation domain. Because of mass conservation, the fluid will flow against the movement of particles at the upper part of the simulation domain, which is called as back flow here. As a result, a recirculation region is observed just above the free surface of the granular flow. In order to minimized the effect of back flows on the runout distance, a large enough domain size for the fluid field is set with length, height and width equal to $0.14 \mathrm{~m}$, $0.06 \mathrm{~m}$ and $0.02 \mathrm{~m}$, respectively.

Fig. 4 shows three cases of the granular flow, including one dry condition: (a) without surrounding fluid; and two wet conditions: (b) in a low-viscosity fluid $\left(\mu_{f}=0.01 \mathrm{~Pa} \cdot \mathrm{s}\right)$ and (c) a high-viscosity fluid $\left(\mu_{f}=0.05 \mathrm{~Pa} \cdot \mathrm{s}\right)$. Compared to the wet condition, it is found that the particles in the dry condition can travel much longer. Part of the particles in the dry condition keep moving forward after the collapse and eventually hit the right wall and bounce back.

In contrast, all the particles are stopped in the wet conditions, because a large part of the kinetic energy of particles is damped by the fluid via drag force. Some energy is turned into fluid kinetic energy and all of it is dissipated due to viscous effects in the end. Considering the granular collapse in a fluid, the drag force on the particles increases as the fluid viscosity increases, which works as a resistant force to prevent the granular column from collapse. Therefore, it takes a longer time for the granular column to collapse and reach its final deposition in the presence of a more viscous fluid. Furthermore, the runout distance is shorter. It is also worth mentioning that the deposit angle of the granular flow increases as the fluid viscosity increases.

The normalized runout distance from LBM-DEM simulations (colored cross symbols) is plotted against the initial aspect ratio in Fig. 5, together with the numerical results from [17] (hollow symbols). It is confirmed that when the aspect ratio and the initial volume fraction are equal to 2 and 0.62 , respectively, both wet conditions in our study belong to the viscous regime [17], in which highviscosity fluids will slow down the granular collapse and cause a reduction in runout distance. 
(a)

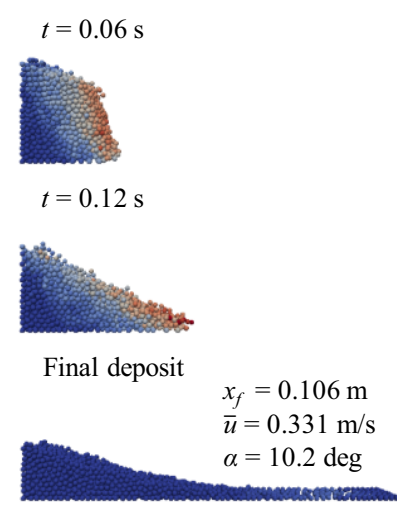

(b)
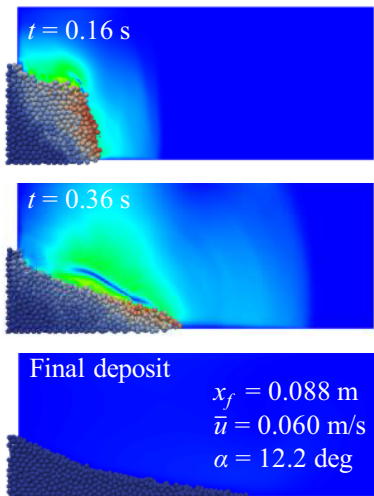

(c)

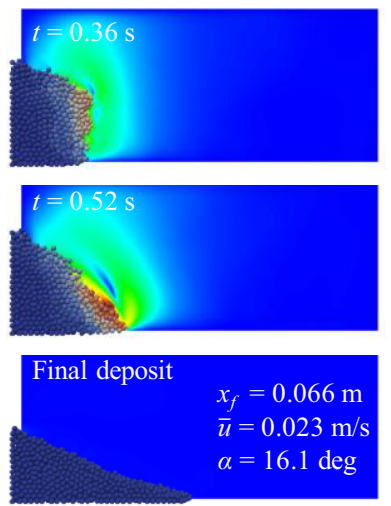

Figure 4. Three sequence of images corresponding to granular particles collapsing on a horizontal plane: (a) granular collapse without surrounding fluid; (b) granular collapse in a fluid with viscosity equal to $0.01 \mathrm{~Pa} \cdot \mathrm{s}$; and (c) $0.05 \mathrm{~Pa} \cdot \mathrm{s}$. The granular and fluid field are coloured by the corresponding velocity

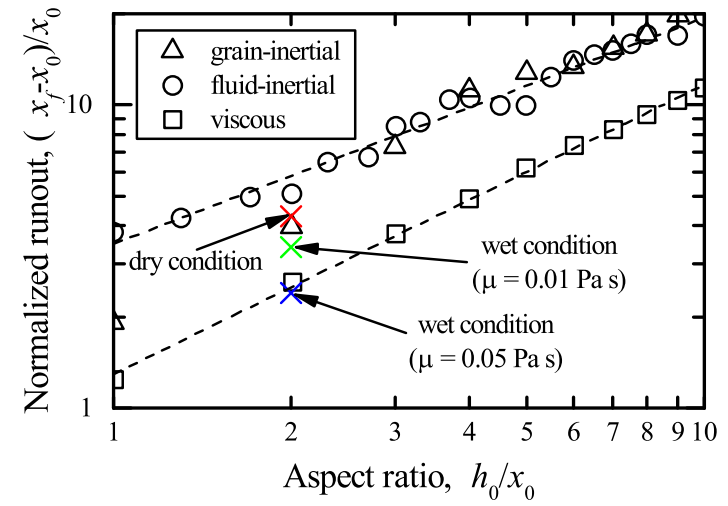

Figure 5. Normalized runout distance as a function of the initial aspect ratio in different regimes referenced in [17]

\section{Conclusions}

A qualitative investigation of the role of fluid viscosity in an immersed granular column is presented using the coupled LBM-DEM. The numerical scheme can successfully capture the hydrodynamic interactions between particle and fluid, which is verified against the available experimental data and analytical solutions. It is found that the presence of fluid slows down the granular flow dramatically. During the collapse of granular column, part of the particle kinetic energy is passed to the fluid through hydrodynamic interactions and finally dissipated inside the fluid due to viscous effects. As a result, when the fluid viscosity increases, the runout distance decreases and the final granular deposit shows a larger deposit angle.

In future works, we plan to fully examine the effect of viscosity and other parameters (for granular material, fluid and composition of granular packing) on the immersed granular collapse quantitatively and determine the appropriate scaling laws governing $x_{f}$ and $h_{f}$. This can be an important risk assessment tool for particle-laden flows and provide optimization scheme for manufacturing industries.

\section{Acknowledgements}

This research is conducted in part using the research computing facilities and/or advisory services offered by Information Technology Services, the University of Hong Kong and under the support of FAP-DF, Brazil.

\section{References}

[1] Lajeunesse, E., J. Monnier, G. Homsy, Phys. Fluids 17, 10 (2005)

[2] Lube, G., Huppert, H. E., Sparks, R. S. J., Phys. Rev. E 72, 4 (2005)

[3] Rondon, L., O. Pouliquen, and P. Aussillous, Phys. Fluids 23, 7 (2011)

[4] Chen, S., G.D. Doolen, Annu. Rev. Fluid Mech. 30, 1 (1998)

[5] He, X., L.-S. Luo, Phys. Rev. E 56, 6 (1997)

[6] Lallemand, P., L.-S. Luo, Phys. Rev. E 61, 6 (2000)

[7] Aidun, C.K., J.R. Clausen, Annu. Rev. Fluid Mech. 42 (2010)

[8] Bhatnagar, P.L., E.P. Gross, M. Krook, Phys. Rev. 94, 3 (1954)

[9] Mei, R., Shyy, W., Yu, D., Luo, L.-S., J. Comput. Phys. 161, 2 (2000)

[10] Cundall, P.A., O.D. Strack, Geotechnique 29, 1 (1979)

[11] Cleary, P.W., Miner. Eng. 11, 11 (1998)

[12] Jing, L., Kwok, C., Leung, Y., Sobral, Y., Phys. Rev. E 94, 5 (2016)

[13] Kloss, C., Goniva, C., Hager, A., Amberger, S., Pirker, S., Prog. Comput. Fluid Dy. 12, 2-3 (2012)

[14] Noble, D., J. Torczynski, Int. J. Mod. Phys. C 9, 8 (1998)

[15] Ten Cate, A., Nieuwstad, C., Derksen, J., Van den Akker, H., Phys. Fluids 14, 11 (2002)

[16] Zick, A., G. Homsy, J. Fluid Mech. 115 (1982)

[17] Topin, V., Monerie, Y., Perales, F., Radjai, F., Phys. Rev. Lett. 109, 18 (2012) 